\title{
Acupuncture for mice
}

Long derided by much of the mainstream medical community, acupuncture seems to have just got a little bit less alternative.

Despite anecdotal evidence claiming benefits in treating ailments from allergies to pain, acupuncture faces two big challenges to acceptance in mainstream medicine. Many reviews of clinical trials have concluded that there is no evidence of efficacy for most conditions beyond the placebo effect ${ }^{1}$, and there is no scientifically accepted mechanism for how the treatment works.

Research in mice has now provided a biochemical explanation that some experts are finding more persuasive ${ }^{2}$, although it might account for only some of the treatment's supposed benefits. "Our study shows there is a clear biological mechanism behind acupuncture," says Maiken Nedergaard, a neuroscientist at the University of Rochester in New York, who led the research.

Nedergaard's team wanted to find out whether the neuromodulator adenosine, which is produced when tissue is injured and has pain-dulling effects, was involved in the purported pain-relieving effects of acupuncture. After inducing pain in the right hind paws of their mice, the researchers inserted and rotated an acupuncture needle just below the 'knee', at a place known in humans as the 'Zusanli point'. For about an hour after the treatment the mice took longer to respond to touch or heat on the paw, indicating that their pain had been dulled. The team found that adenosine levels had increased at the acupuncture site, and that mice lacking a key cell receptor for adenosine did not show the same response.

"One thing that's really nice about this is they approach this question with a specific and firm hypothesis," says Vitaly Napadow, a neuroscientist who studies acupuncture at Harvard Medical School in Boston, Massachusetts. Although sceptical that the mechanism could explain, for example, how acupuncture could relieve headaches, he says that "in conditions such as carpal tunnel syndrome, a mechanism such as that described in this paper might very well be important".

Dominik Irnich, head of the Multidisciplinary Pain Centre at the University of Munich in Germany, and a doctor who uses acupuncture, notes that other studies have proposed mechanisms such as the release of endorphins or other neurotransmitters ${ }^{3,4}$. But Nedergaard says that these would act on the whole nervous system - her study found no effect when acupuncture was applied to the rodents' pain-free left legs, suggesting that there is not a central mechanism.

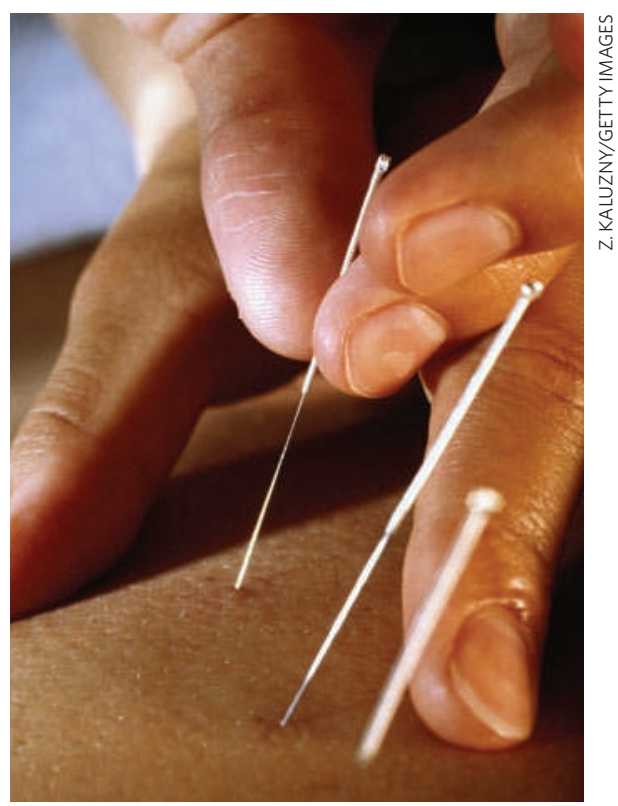

Is acupuncture more than mere placebo?

Edzard Ernst, who studies the effectiveness of alternative therapies at the Peninsula Medical School in Exeter, UK, says that the mechanism is credible, but that the work does not address whether acupuncture is an effective treatment. "If the clinical effect is not beyond placebo, which most of the well-controlled clinical trials seem to suggest, the mechanism is irrelevant and the true mechanism is placebo," he says.

Jana Sawynok, a pharmacologist who studies the pain-modulating effects of adenosine at Dalhousie University in Halifax, Canada, notes that caffeine blocks the adenosine receptor pinpointed in this study. Given the caffeine intake of many countries where acupuncture trials are carried out, this could be a serious confounding issue in trials, she suggests.

Nedergaard says her work may open the way to making acupuncture more effective. Her study also treated the mice with a drug called deoxycoformycin, which suppresses the breakdown of adenosine and is approved in the United States for treating some types of leukaemia. The drug prolonged the pain-relieving effects of the acupuncture treatment by more than an hour; Nedergaard is now trying to organize a trial of this strategy in humans.

Daniel Cressey

1. Ernst, E. J. Pain Symptom Manage. 37, 709-714 (2009).

2. Goldman, N. et al. Nature Neurosci. doi:10.1038/nn.2562 (2010).

3. Clement-Jones, V. et al. Lancet 316, 946-949 (1980).

4. Bing, Z. et al. Pain 47, 71-77 (1991). 